\title{
Computational Modeling of Dynamic Stability Derivatives for Generic Airfoils
}

\author{
Muhammad Saleem Mumtaz, Adnan Maqsood and Salma Sherbaz \\ Research Center for Modeling \& Simulation (RCMS), National University of Sciences and Technology (NUST), H-12 Campus, Islamabad, \\ Pakistan
}

\begin{abstract}
This paper presents a method for the computation of the static and dynamic stability derivatives of generic airfoils using high fidelity Computational Fluid Dynamics. Aerodynamic coefficients are calculated for NACA 0012 airfoil and flat plate at different angles of attack. Results of lift coefficient are validated with experimental data. Static and dynamic stability derivatives are calculated by oscillating the airfoil geometry at suitable frequency. Simulations are performed at various flight conditions in terms of angles of attack, frequencies and oscillation amplitudes. The aim of the work is to decipher the behaviour of longitudinal damping derivatives used in flight mechanics through CFD. This approach enables the efficient and accurate computation of dynamic derivatives. Calculations are done for constant air velocity altering only the angle of attack. Inviscid model is tested since its results nearly match with experimental data. The simulations show that the nonlinear characteristics of the stability derivatives are captured by varying angle of attack.
\end{abstract}

\section{Introduction}

Design cycle of modern aircraft has reduced significantly during recent years, partly due to introduction of computers on various stages of design. Complexity in the design of manned and unmanned vehicles is gradually introduced for improved manoeuvrability and versatile mission requirements, which increases the requirement for precise guess of aerodynamic coefficients and dynamic stability derivatives during the preliminary design stage. In 1911 concept of these dynamic derivatives was given by Byran[1].Different mathematical models use these dynamic derivatives to calculate moments and aerodynamic forces of aircrafts. These aerodynamics derivatives and moments are functions of aircraft states. It is easy to predict static stability derivative than dynamic derivative. Conventionally, wind tunnel [2-9] tests were performed to measure the derivatives. For static stability derivatives wind tunnel test give good estimation but due to complex test-setup, fast design modification and time to perform these experiment, other methods such as DATCOM [10], and ESDU [11] are used. These two methods are fast compared to wind tunnel. However, these techniques may have question for accuracy. In nonlinear system [12-15] importance of dynamic derivatives has increased due to flight manoeuvres by UAVs and other flight vehicles. in recent times, Computational Fluid Dynamics (CFD) is used as an another tool to guess aerodynamic stability derivatives. Computational Fluid Dynamic has the potential to replace the wind tunnel tests and experiential methods as it is inexpensive with minimum physical and kinematical limitations. Many researchers have used CFD to calculate stability derivatives due to these advantages. In this paper sensitivity of amplitude and frequencies on dynamic stability in pitch is quantified. For validation purpose NACA 0012 airfoil is used. Initially normal force coefficient for this airfoil is calculated and is compared with experimental data. From the momentalpha graph longitudinal derivatives for airfoil are calculated. Using the same technique, these derivatives are calculated for flat plate.

\section{Geometry and meshing}

For the current study, NACA0012 airfoil is chosen as the validation platform. The coordinates of the profile are produced into GAMBIT ${ }^{\circledR}$. The chord length of the airfoil is $1 \mathrm{~m}$. The center of gravity was chosen as $0.25 \mathrm{c}$ for calculation of lift, drag and moment coefficients. For steady state analysis, airfoil remains at rest at zero degree angle of attack. The computational model with mesh for analysis is shown in Figure 1.
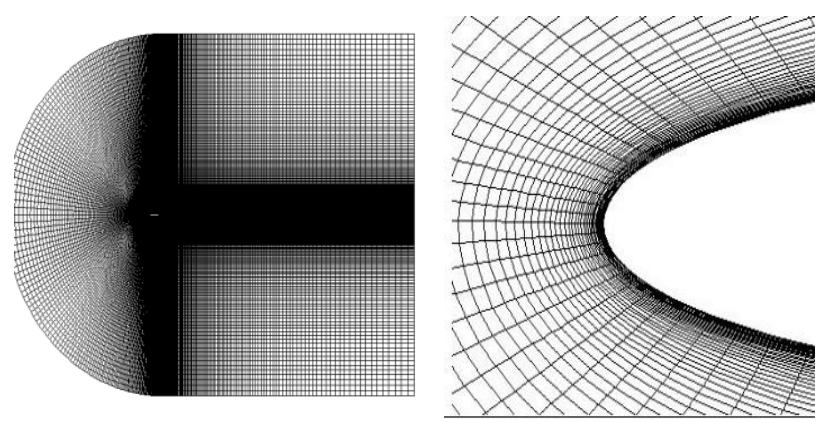

Figure 1. Mesh around airfoil. 


\section{NACA 0012 Airfoil studies}

\subsection{Steady state analysis}

For steady state analysis, pre-processing is done in GAMBIT ${ }^{\circledR}$. These files are exported in FLUENT ${ }^{\circledR}$ to solve the governing equations of fluid mechanics. For validation with experimental data, steady-state analysis is done by using different turbulence models and for different angles of attack. Parameters for this analysis are taken from AGARD 702 [16] report.

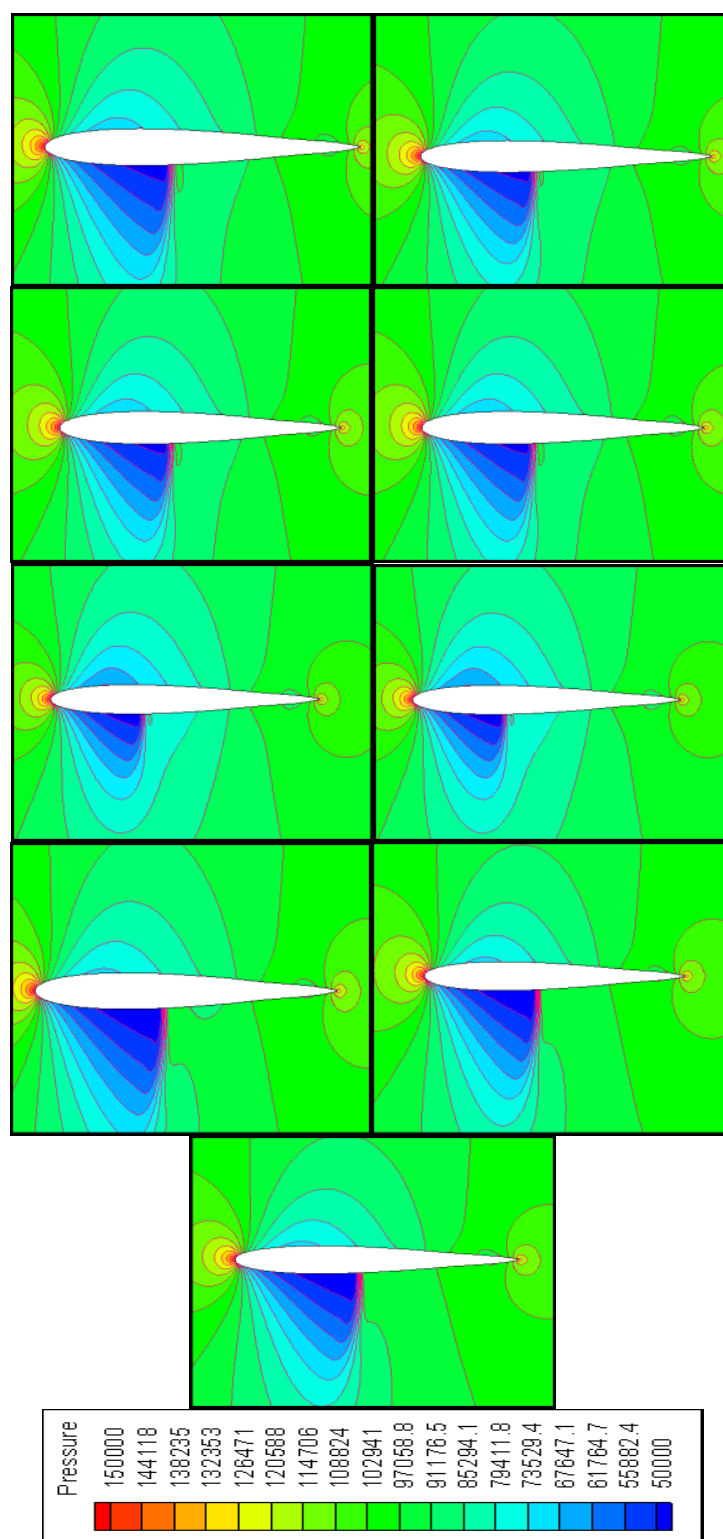

Figure 2. Pressure Contours for frequencies 32.66, 42.66, 52.66 and amplitude 1.51, 2.51 and 3.51 .

When simulation for this analysis is converged, lift, drag and moment coefficients are calculated. From these coefficients, normal force coefficient is calculated and is compared with experimental data using following equation.

$$
C_{N}=C_{l} \cos (\alpha)+C_{d} \sin (\alpha)
$$

where $C_{N}$ and $\alpha$ represent normal force coefficient and angle of attack respectively. Lift and drag coefficient convergence histories for steady state analysis at zero degree angle of attack using inviscid model are done. Pressure contours for NACA 0012 airfoil at different amplitudes, frequencies and at zero angle of attack are plotted in the Figure 2.

Results obtained for normal force coefficient are compared with experimental data in Figure 3.

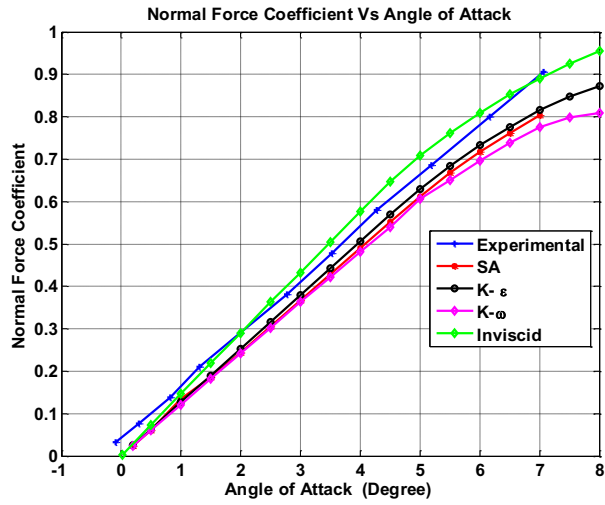

Figure 3. Normal force coefficient comparisons.

\subsection{Unsteady analysis}

For unsteady analysis, sinusoidal pitching motion of airfoil is achieved by applying dynamic mesh technique. Schematic of airfoil pitching motion is shown in Figure 4. A user define function is made in c language which is compiled in Fluent ${ }^{\circledR}$ that control the dynamic motion of airfoil. A domain in circular form is made around the airfoil as an interface. This interface oscillates along with airfoil with a suitable angular frequency. Motion of airfoil is along z-axis. The oscillating point of airfoil is $0.25 \mathrm{c}$. The function made for oscillation is as follows

$$
\alpha(t)=\alpha 0+\alpha l \sin (\omega t)
$$

where $\alpha_{0}=$ Initial angle of attack, $\alpha_{1}=$ Amplitude of oscillation and $\omega=$ Angular frequency.

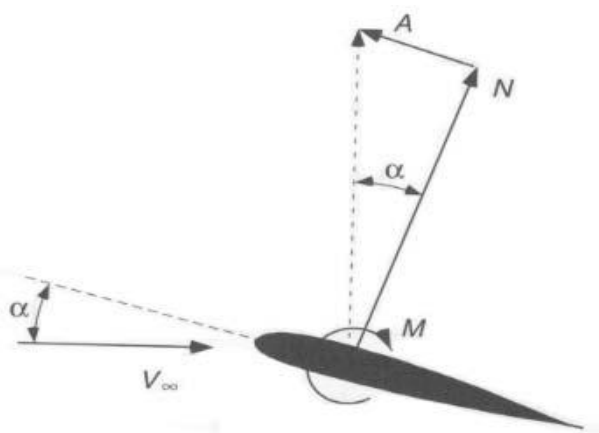

Figure 4. Airfoil pitching motion.

\section{Methodology}

Procedure for calculating dynamic derivative is depicted in Figure 5. 


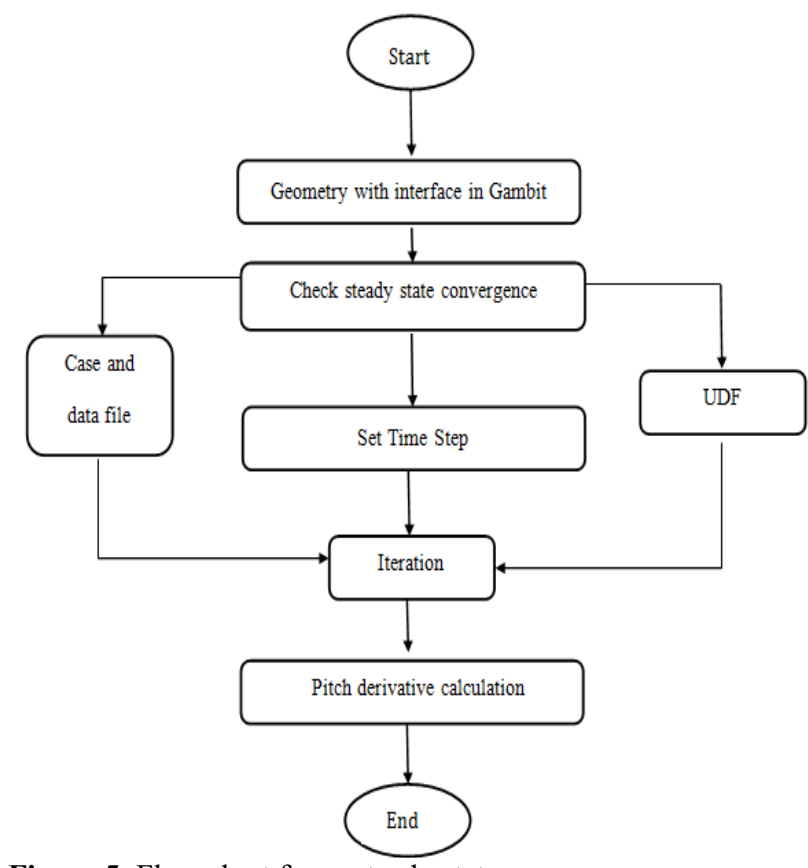

Figure 5. Flow chart for unsteady state.

Analysis parameters for unsteady analysis are also taken from AGARD 702 report and are given in the Table 1.

Table 1. Unsteady analysis parameters.

\begin{tabular}{|c|c|c|}
\hline No & Parameters & Values \\
\hline 1 & Airfoil & NACA0012 \\
\hline 2 & Mach $(\mathrm{M})$ & 0.755 \\
\hline 3 & Initial angle of attack & 0.016 degree \\
\hline 4 & Amplitude & 2.51 \\
\hline 5 & Pitch center & $0.25 \mathrm{c}$ \\
\hline 6 & Reduced Frequency & 0.0814 \\
\hline 7 & Angular Velocity & $42.66 \mathrm{rad} / \mathrm{s}$ \\
\hline
\end{tabular}

Lift coefficient profiles for unsteady case are plotted below in Figure 6. Result of the current study is validated in the form of lift coefficient with experimental data which shown in Figure 7.

In aerodynamics of flight vehicles, stability plays an important role. In order to approximate dynamic stability, damping derivative calculation is necessary. These derivatives have three types, pitch, yaw and roll. Here we have calculated the pitch damping derivatives of 2-D NACA0012 airfoil. Pitch Derivatives are calculated using the following relation.

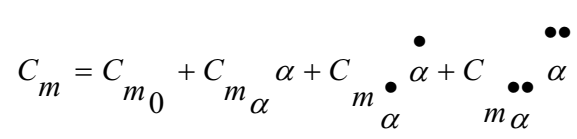

Neglecting higher value of derivatives, Eq (3) will reduce to

$$
C_{m} \approx C_{0}+C_{\alpha} \alpha+C m_{\alpha}^{\bullet} \stackrel{\bullet}{\bullet}
$$

Pitch derivatives for airfoil using different frequency and amplitude are calculated in the current study.In order to calculate pitch derivatives coefficients are related to oscillating parameters using time as variable. In order to determine pitch derivatives relation shown in figure 8 will helps us. The trend of the graph between the lift coefficient and the angle of attack is linear. There is shown a distinct hysteresis in the solution, as verified by the gap between the coefficient on the upstroke and down stroke of the oscillation.

This hysteresis helps us to find a dot or transient derivatives. Transient derivatives with respect to

$\dot{\alpha}$ derivatives, transient derivatives represent the time lag in the development of the these coefficient resulting from a change in the flow as discussed by Etkin [17]. This idea helps us to compute the stability derivatives. To calculate stability derivatives from this solution, we compute linear least-squares fit of the output coefficient $C_{L}$ with respect to $\alpha$.

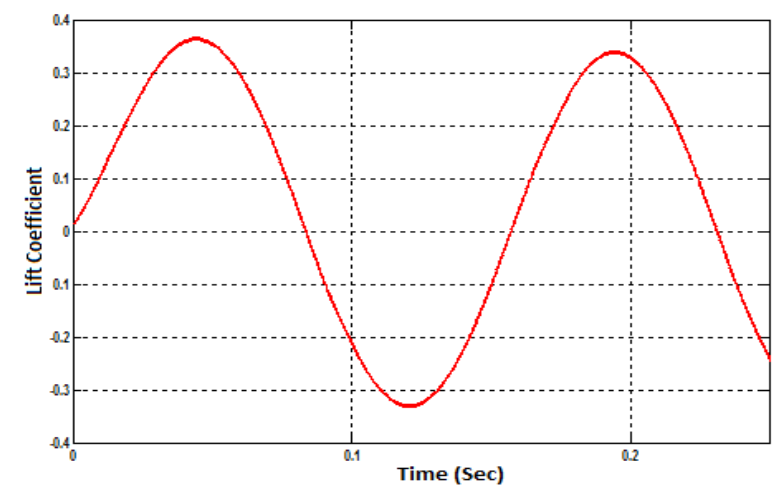

Figure 6. Lift coefficient profile.

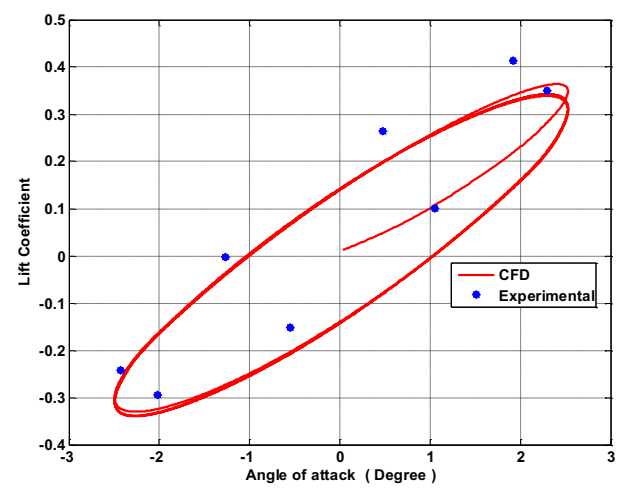

Figure 7. Comparison of lift coefficient.

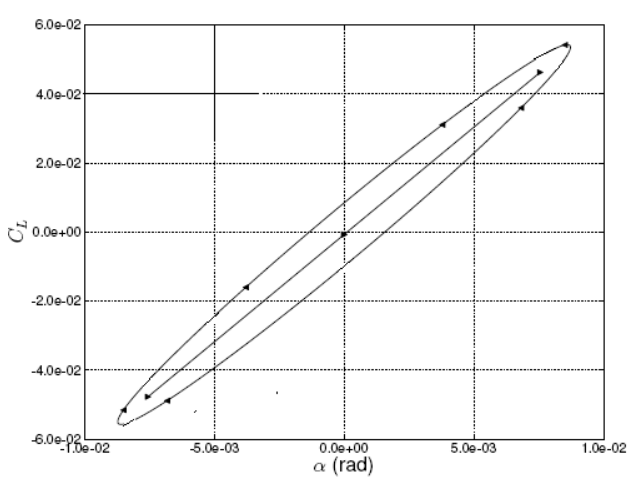

Figure 8. $C_{L}$ vs $\alpha$. 
In the Figure 8 the stability derivative $\left(C_{L_{\alpha}}\right)$ is calculated by the slope which is obtained by least square fit method. Y-intercept of the line is the value of the lift coefficient $\left(C_{L}\right)$ at the zero value of the motion perturbation $\Delta_{\alpha}=0$. From this an equation of the following form is obtained

$$
y=C_{i} x+C_{i_{0}}
$$

From this hysteresis transient derivatives can be calculated. This quantity is obtained by subtracting the value of linear recession line from the time spectral solution

$$
R_{c_{i}}^{n}=C_{i}^{n}-y\left(x^{n}\right)
$$

From above equation another strongly linear relationship is obtained, as shown in Figure 9.

The slope of above graph gives the transient derivative $\left(C_{l_{\alpha}}^{\bullet}\right)$. Using this technique Pitch damping derivative $\left(C_{m_{\alpha}}^{\bullet}\right)$ is calculated for the current study. Results obtained for different amplitude and at different frequencies are given in Table 2 .

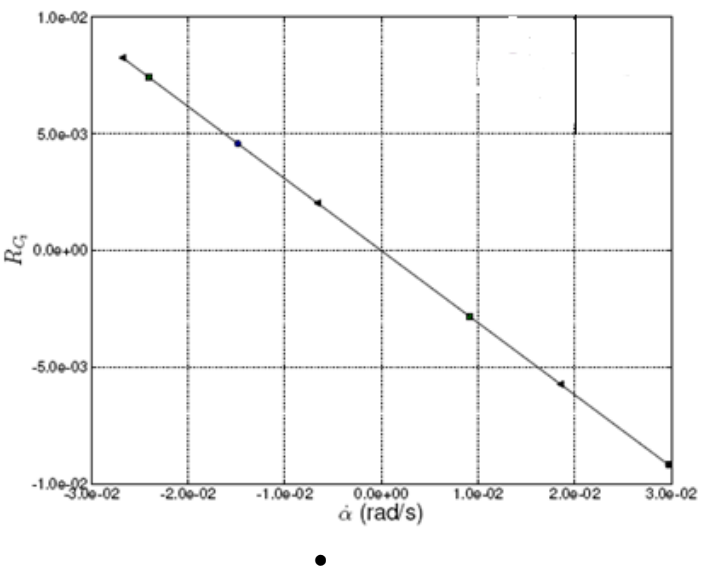

Figure 9. $R_{c_{i}}$ Hysteresis vs $\dot{\alpha}$.

\begin{tabular}{|c|c|c|c|c|c|c|}
\hline \multirow{3}{*}{ } & \multicolumn{6}{|c|}{ Frequency } \\
\hline & \multicolumn{2}{|c|}{$\omega=32.66$} & \multicolumn{2}{|c|}{$\omega=42.66$} & \multicolumn{2}{|c|}{$\omega=52.66$} \\
\hline & $\operatorname{Cm\alpha }$ & $\mathrm{Cm} \alpha^{\circ}$ & $\operatorname{Cm} \alpha$ & $\mathrm{Cm} \alpha^{\circ}$ & $\operatorname{Cm\alpha }$ & $\mathrm{Cm} \alpha^{\circ}$ \\
\hline 1.51 & 0.002 & -0.006 & 0.002 & -0.006 & 0.002 & -0.005 \\
\hline 2.51 & 0.001 & -0.006 & 0.001 & -0.006 & 0.001 & -0.0056 \\
\hline 3.51 & 0 & -0.005 & 0 & -0.005 & 0 & -0.0046 \\
\hline
\end{tabular}

Table 2. Pitch damping derivatives.

\section{Flat plate geometry}

Procedure for calculation of pitch derivative for NACA 0012 airfoil is also applied for flat plat e. Geometry for flat plate is shown in Figure 10.
Figure 10. Flat Plate Geometry.

Pitch derivatives are calculated at frequency $(\mathrm{f}=42.66$ $\mathrm{rad} / \mathrm{sec})$ and amplitude $(\mathrm{a}=2.51 \mathrm{~m})$ for different angles of attack. Results obtained are given in table 3 and are plotted in Figure 11.

Table 3. Pitch derivatives for Flat Plate.

\begin{tabular}{|l|l|l|}
\hline Angle (Deg.) & $\mathbf{C m \alpha}$ & $\mathbf{C m} \boldsymbol{\alpha}^{\cdot}$ \\
\hline 0 & 0.002 & -0.008 \\
\hline 2 & 0.003 & -0.010 \\
\hline 4 & -0.003 & -0.004 \\
\hline 6 & -0.010 & 0.010 \\
\hline 8 & -0.04 & -0.074 \\
\hline
\end{tabular}

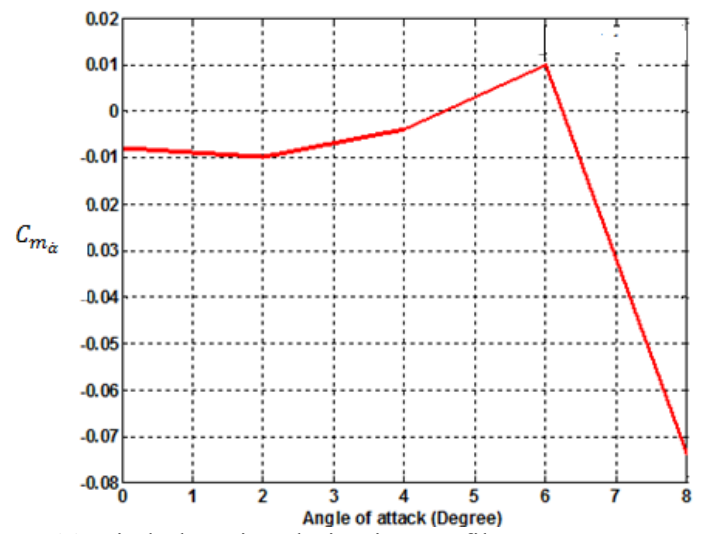

Figure 11. Pitch damping derivative profile.

\section{Conclusion}

The results obtained from the current simulation for NACA 0012 and for the Flat plate show good agreement with experimental data obtained from wind tunnel test and the data obtained from literature. The response of different parameters like angle of attack, angular frequency, amplitude of oscillation, time step, and turbulence model and gap variation between the flat plates was also studied. After analysis of computational data obtaining form the simulation of NACA 0012 airfoil, it was found that for the same value of angular frequencies and for increased value of oscillating amplitude the value of pitch damping derivative increases in other words we can say that dynamic stability decrease. For flat plate point of view different simulations were performed at fixed amplitude and at fixed angular frequency. From the result data it was found that by increasing the angle of attack to 6 degree pitch damping derivative increases and further increase in angle of attack its value decreases.

\section{References}

1. G. H. Bryan, Stability in aviation. London, UK: Macmillan Publishers, (1911). 
2. I. Shantz and R. T. Groves, "Dynamic and static stability measurements of the basic finner at supersonic speeds," (1960).

3. L. C. MacAllister, "The Aerodynamic Properties of a Simple Non Rolling Finned Cone-Cylinder Configuration Between Mach Numbers 1.0 and 2.5," (1955).

4. G. L. Winchenbach, B. L. Uselton, and R. M. Chelekis, J. Aircr., 21, 1, pp. 5-13, (1984).

5. B. L. Uselton, "A description of the Standard Dynamic Model (SDM)," in $56^{\text {th }}$ Supersonic Tunn. Assoc. Meet., (1981).

6. N. Alemdaroglu, I. Iyigun, M. Altun, H. Uysal, F. Quagliotti, and G. Guglieri, "Determination of dynamic stability derivatives using forced oscillation technique," in $40^{\text {th }}$ AIAA Aerosp. Sci. Meet. Exhib., American Institute of Aeronautics and Astronautics, Reno, NV, USA, (2002).

7. G. Guglieri, F. Quagliotti, and P. Scarabelli, "Static and Oscillatory Experiments on the SDM at Politecnico di Torino," Nota Sci. E Tec., no. 74/93, (1993)

8. A. R. Davari and M. R. Soltani, Iran. J. Sci. Technol., 31, B1, pp. 49, (2007).

9. E. Schmidt, "Standard dynamics model experiments with the DFVLR/AVA transonic derivative balance," Institut fuer aeroelastik goettingen (1985).

10. Engineering Sciences Data Unit (ESDU sheets), www.esdu.com.
11. R. Hall, R. Biedron, D. Ball, D. Bogue, J. Chung, B. Green, M. Grismer, G. Brooks, and J. Chambers, "Computational Methods for Stability and Control (COMSAC): The Time Has Come," in AIAA Atmospheric Flight Mechanics Conference and Exhibit, American Institute of Aeronautics and Astronautics, (2005).

12. Maqsood, A. and Go, T. H., 'Multiple time scale analysis of aircraft longitudinal dynamics with aerodynamic vectoring' Nonlinear Dynamics, 69, 3, pp. 731-742, (2012).

13. Maqsood, A. and Go, T. H., 'Longitudinal Flight Dynamic Analysis of an Agile UAV' Aircraft Engineering and Aerospace Technology, 82, 5, pp. 288-295, (2010).

14. Go, T. H. and Maqsood, A. , 'Effect of aspect ratio on wing rock at low Reynolds number' Aerospace Science and Technology, 42, pp. 267-273, (2015).

15. Bibi, A., Maqsood, A. and Go, T. H., 'Review on Analysis \& Modeling of Dynamic Stability Characterisitics of Atmospheric Entry Vehicles' NUST Journal of Engineering Sciences, 7, 1, pp. 1521, (2015).

16. R. H. Landon, "Compendium of unsteady aerodynamic measurements," AGARD Rep., 702, (1982).

17. B. Etkin, Dynamics of Atmospheric Flight, Dover Publications, Mineola, New York. 